\title{
THE DISTRIBUTION OF THE RESIDUES OF A QUARTIC POLYNOMIAL
}

\author{
by $\mathrm{K}$. MCCANN AND K. S. WILLIAMS
}

(Received 17 June, 1966)

1. Introduction. Let $f(x)$ denote a polynomial of degree $d$ defined over a finite field $k$ with $q=p^{n}$ elements. B. J. Birch and H. P. F. Swinnerton-Dyer [1] have estimated the number $N(f)$ of distinct values of $y$ in $k$ for which at least one of the roots of

$$
f(x)=y
$$

is in $k$. They prove, using A. Weil's deep results [12] (that is, results depending on the Riemann hypothesis for algebraic function fields over a finite field) on the number of points on a finite number of curves, that

$$
N(f)=\lambda q+O\left(q^{\frac{1}{2}}\right),
$$

where $\lambda$ is a certain constant and the constant implied by the $O$-symbol depends only on $d$. In fact, if $G(f)$ denotes the Galois group of the equation (1.1) over $k(y)$ and $G^{+}(f)$ its Galois group over $k^{+}(y)$, where $k^{+}$is the algebraic closure of $k$, then it is shown that $\lambda$ depends only on $G(f), G^{+}(f)$ and $d$. It is pointed out that " in general "

$$
\lambda=1-\frac{1}{2 !}+\frac{1}{3 !}-\ldots-(-1)^{d} \frac{1}{d !} \text {. }
$$

It is the purpose of this paper to consider the case of quartic polynomials $(\bmod p)($ so that $d=4$ and $q=p$ ) in greater detail. It is shown, using Skolem's work [9] on the general quartic polynomial $(\bmod p)$ and Manin's elementary proof [5] of Hasse's result

$$
\left|\sum_{x=0}^{p-1}\left(\frac{x^{3}+a x+b}{p}\right)\right|<2 p^{t}
$$

that (1.2) can be proved in this special case in a completely elementary way, which incidently avoids explicit consideration of $G(f)$ and $G^{+}(f)$. Further it is shown that the only values of $\lambda$ which occur are

$$
\lambda=\frac{5}{8}\left(=1-\frac{1}{2 !}+\frac{1}{3 !}-\frac{1}{4 !}\right), \frac{1}{2}, \frac{3}{8}, \frac{1}{4} ;
$$

and moreover it is determined when each of these occurs. For those $f$ having $\lambda=\frac{1}{2}, \frac{3}{8}$ or $\frac{1}{4}$, it is proved that the error term in the asymptotic formula for $N(f)$ is in fact $O(1)$. In the case of cubic polynomials [6] the corresponding values of $\lambda$ are

$$
\lambda=1, \quad \frac{2}{3}(=1-1 / 2 !+1 / 3 !), \quad \frac{1}{3} ;
$$

$\mathbf{E}$ 
and in this case the error term is always $O(1)$. We note that for cubic and quartic polynomials, the number of $\lambda$-values occurring is the same as the degree of the polynomial under consideration. We also observe that for $d=3$ and 4

$$
f^{*}(x, y)=\frac{f(x)-f(y)}{x-y}
$$

is absolutely irreducible $(\bmod p)$ if and only if

$$
\lambda=1-\frac{1}{2 !}+\frac{1}{3 !}-\ldots-(-1)^{d} \frac{1}{d !} .
$$

(For $d=3$ this was first noted by S. Uchiyama [10].)

We also consider the problem of determining the number of residues in an arithmetic progression. If the arithmetic progression has $h$ terms we prove that the number of residues in it is given by

$$
\lambda h+O\left(p^{\frac{1}{2}} \log p\right),
$$

where $\lambda$ is given by (1.3) and the constant implied by the $O$-symbol is absolute. This proves that any arithmetic progression with $\gg p^{\frac{1}{2}} \log p$ terms contains a residue of $f(x)(\bmod p)$, generalizing a result of L. J. Mordell [7] in the case $d=4$. It is shown that it also contains a non-residue (generalizing a result of one of us [14]) and a pair of consecutive residues. (Similar results have been shown to hold in the cubic case [6].) This last result verifies a conjecture of one of us [13] in a special case, namely, that the least pair of consecutive nonnegative residues of any polynomial $(\bmod p)$ of degree $d$ is $O\left(p^{\frac{1}{2}} \log p\right)$.

Finally we conjecture that (1.4) holds for all polynomials of degree $d$. The truth of this conjecture would imply that the least non-negative non-residue $(\bmod p)$ of a polynomial of degree $d$, for which $\lambda \neq 1$, is $O\left(p^{\frac{1}{2}} \log p\right)$.

2. Simplification of the problem. Let

$$
f_{1}(x)=a_{1} x^{4}+b_{1} x^{3}+c_{1} x^{2}+d_{1} x+e_{1} \quad\left(a_{1} \neq 0\right) \dagger
$$

have the $N$ residues $(\bmod p)$

$$
r_{1}, r_{2}, \ldots, r_{N}
$$

Then

where

$$
f_{2}(x)=x^{4}+b_{2} x^{3}+c_{2} x^{2}+d_{2} x+e_{2},
$$

$$
b_{2}=a_{1}^{-1} b_{1}, \quad c_{2}=a_{1}^{-1} c_{1}, \quad d_{2}=a_{1}^{-1} d_{1}, \quad e_{2}=a_{1}^{-1} e_{1},
$$

also has $N$ residues, namely

$$
a_{1}^{-1} r_{1}, a_{1}^{-1} r_{2}, \ldots, a_{1}^{-1} r_{N}
$$

$\dagger$ Very often we omit $(\bmod p)$ as this is the only modulus occurring. 
Now let

$$
f_{3}(x)=f_{2}\left(x-4^{-1} b_{2}\right)=x^{4}+c_{3} x^{2}+d_{3} x+e_{3},
$$

so that

and

$$
c_{3}=-2^{-3} \cdot 3 b_{2}^{2}+c_{2}, \quad d_{3}=2^{-3} b_{2}^{3}-2^{-1} b_{2} c_{2}+d_{2}
$$

$$
e_{3}=-3.2^{-8} b_{2}^{4}+2^{-4} b_{2}^{2} c_{2}-2^{-2} b_{2} d_{2}+e_{2} .
$$

Then $f_{3}(x)$ also has the $N$ residues (2.1). Now set

The residues of $f_{4}(x)$ are

$$
f_{4}(x)=f_{3}(x)-e_{3} \text {. }
$$

$$
a_{1}^{-1} r_{1}-e_{3}, a_{1}^{-1} r_{2}-e_{3}, \ldots, a_{1}^{-1} r_{N}-e_{3} .
$$

Hence, without loss of generality, we need only consider the number of residues $(\bmod p)$ of

$$
f(x)=x^{4}+a x^{2}+b x \text {. }
$$

When we count the residues $(\bmod p)$ only if they lie in a certain arithmetic progression, say

$$
\{l+m s\} \quad(s=0,1, \ldots, h-1),
$$

we can still work with (2.2) without any loss of generality, as the formula obtained for the number of its residues in (2.3) is of the form

$$
\lambda h+O\left(p^{\frac{1}{2}} \log p\right),
$$

where $\lambda$ is the constant discussed in $\$ 1$ and the constant implied by the $O$-symbol is absolute $\dagger$ and so does not depend on $l$ and $m$.

Throughout this paper we will use the following notation. We let $N_{r}(r=0,1,2, \ldots, p-1)$ denote the number of incongruent $(\bmod p)$ solutions $x$ of

and set

$$
f(x) \equiv r(\bmod p),
$$

$$
n_{i}=\sum_{N_{r}=i}^{r} 1 \quad(i=0,1,2,3,4)
$$

where the summation in $r$ is taken over the set $\{0,1,2, \ldots, p-1\}$. The number $N(f)$ of residues of $f(x)$ is therefore just

$$
\sum_{\substack{r \\ N_{r}>0}} 1=n_{1}+n_{2}+n_{3}+n_{4} .
$$

For the residues of $f(x)(\bmod p)$ in the arithmetic progression $(2.3)$, we let $M(f)$ denote their number and introduce

$$
m_{i}=\sum_{\substack{r \\ N_{r}=i}}^{\prime} 1 \quad(i=0,1,2,3,4),
$$

† Unless otherwise stated; all constants implied by $O$-symbols are absolute. 
where the dash (') denotes that the summation in $r$ is taken over the set (2.3). Hence

$$
M(f)=m_{1}+m_{2}+m_{3}+m_{4} .
$$

3. Estimation of $n_{3}$. The discriminant of $f(x)-r$ is given by

$$
D(r)=-256 r^{3}-128 a^{2} r^{2}-\left(16 a^{4}+144 a b^{2}\right) r-\left(4 a^{3} b^{2}+27 b^{4}\right) .
$$

Hence $D(r) \equiv 0(\bmod p)$ has at most three incongruent solutions $r$, that is $f(x)-r$ has a squared factor $(\bmod p)$ for $O(1)$ values of $r$. But $N_{r}=3$ implies that $f(x)-r$ has a squared linear factor $(\bmod p)$, and so we have

LeMMA 1. $n_{3}=O(1)$.

4. Estimation of $n_{1}$. If $b \equiv 0$, obviously $n_{1}=O(1)$ so that we may suppose that $b \neq 0$. The cubic resolvent of $f(x)-r$, having the same discriminant as $f(x)-r$, apart from a factor $2^{12}$, is

$$
g_{r}(y)=y^{3}+8 a y^{2}+16\left(a^{2}+4 r\right) y-64 b^{2} .
$$

Now, by a result of Skolem [9], $f(x)-r$ is congruent to the product of a linear polynomial and an irreducible cubic $(\bmod p)$ if and only if $g_{r}(y)$ is irreducible $(\bmod p)$. Hence

$$
n_{1}=\sum_{\substack{r \\ \text { irred }(\bmod p)}}+O(1)
$$

or equivalently

$$
n_{1}=p-\sum_{\substack{r \\ g r \operatorname{red}(\bmod p)}} 1+O(1)
$$

As discrim $g_{r}(y)=2^{12} D(r)$, there are at most three values of $r$ for which $g_{r}(y)$ has a squared factor $(\bmod p)$. Let $n^{(1)}$ denote the number of $r$ for which $g_{r}(y)$ has exactly one linear factor and $n^{(3)}$ the number of $r$ for which $g_{r}(y)$ has three distinct linear factors $(\bmod p)$. Then

$$
n_{1}=p-\left(n^{(1)}+n^{(3)}\right)+O(1) \text {. }
$$

Now

$$
n^{(1)}+3 n^{(3)}=p+O(1)
$$

so that

$$
n_{1}=\frac{2}{3} p-\frac{2}{3} n^{(1)}+O(1)
$$

Now $g_{r}(y)$ has exactly one linear factor if and only if

$$
\left(\frac{\operatorname{discrim} g_{r}(y)}{p}\right)=-1 \text {. }
$$


This was first proved by L. E. Dickson [4]. Hence

$$
\begin{aligned}
n^{(1)} & =\frac{1}{2} \sum_{r}\left\{1-\left(\frac{D(r)}{p}\right)\right\}+O(1) \\
& =\frac{1}{2} p+O\left(p^{\frac{1}{2}}\right),
\end{aligned}
$$

by Manin's result [5]. Hence we have proved in an elementary way

Lemma 2.

$$
n_{1}= \begin{cases}\frac{1}{3} p+O\left(p^{\frac{1}{2}}\right), & \text { if } \quad b \neq 0, \\ O(1), & \text { if } b \equiv 0\end{cases}
$$

5. Estimation of $n_{2}$. In this section we give two different proofs of our estimates for $n_{2}$. The first proof appears to be deep but is easily generalized to deal with $m_{2}$. The second proof is elementary and completes the elementary proof of the asymptotic formula for $N(f)$. This method does not seem to be easily capable of generalization to $m_{2}$. To calculate $m_{2}$ by this method would require an asymptotic formula for $m_{1}+4 m_{2}+9 m_{3}+16 m_{4}$, which, after applying the method of incomplete sums to it, requires an effective estimate for

$$
\max _{1 \leqq 0 \leqq p-1}\left|\sum_{\substack{x, y=0 \\ f(x) \equiv f(y)}}^{p-1} e(-v f(y))\right|,
$$

where, for any real $t, e(t)$ denotes $\exp \left(2 \pi i t p^{-1}\right)$. Such an estimate seems difficult to obtain.

First Proof. We consider two cases according as $b \equiv 0$ or $b$ = 0 .

Case (i): $b \equiv 0$. In this case

$$
f(x)-r \equiv x^{4}+a x^{2}-r
$$

is congruent to the product of an irreducible quadratic and two distinct linear factors if and only if

$$
\left(\frac{-r}{p}\right)=-1 \text { and }\left(\frac{4 r+a^{2}}{p}\right)=+1
$$

This result is contained in a theorem of Carlitz [2]. (Skolem [9] seems to forget the possibility $a_{1}^{3}-4 a_{1} a_{2}+8 a_{3} \equiv 0$ (his notation) in his paper; in our case we have $a_{1}=0, a_{2}=a, a_{3}=0$ and $a_{4}=-r$.) Hence

$$
\begin{aligned}
n_{2} & =\frac{1}{4} \sum_{r}\left\{1-\left(\frac{-r}{p}\right)\right\}\left\{1+\left(\frac{4 r+a^{2}}{p}\right)\right\}+O(1) \\
& =\frac{1}{4}\left\{p-\sum_{r}\left(\frac{-4 r^{2}-a^{2} r}{p}\right)\right\}+O(1) \\
& =\frac{1}{4}\left\{-p\left(\frac{-1}{p}\right)\left[p\left(1-\left(\frac{a^{2}}{p}\right)\right)-1\right]\right\}+O(1) \\
& =\frac{1}{4}\left\{1-\left(\frac{-1}{p}\right)\left[1-\left(\frac{a^{2}}{p}\right)\right]\right\} p+O(1) .
\end{aligned}
$$


Case (ii): $b$ 丰 0 . In this case

$$
f(x)-r=x^{4}+a x^{2}+b x-r
$$

is congruent to the product of an irreducible quadratic and two linear distinct factors if and only if

$$
g_{r}(y) \equiv\left(y-y_{1}\right) h_{r}(y) \quad\left(y_{1} \equiv y_{1}(r)\right),
$$

where $h_{r}(y)$ is an irreducible quadratic and $\left(y_{1} \mid p\right)=+1$; for convenience we occasionally use this alternative notation for Legendre symbols.

Now $g_{r}(y)$ is of the form (5.1) if and only if

$$
\left(\frac{\operatorname{discrim} g_{r}(y)}{p}\right)=-1 \text {, }
$$

i.e., if and only if

$$
\left(\frac{D(r)}{p}\right)=-1
$$

Hence

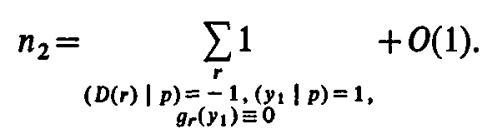

As $D(r)$ is a cubic in $r$, the number of $r$ with $(D(r) \mid p)=-1$ is just

$$
\frac{1}{2} \sum_{r}\left\{1-\left(\frac{D(r)}{p}\right)\right\}+O(1)=\frac{1}{2} p+O\left(p^{\frac{1}{4}}\right)>0,
$$

for large enough $p$.

Hence there exists at least one $r$ such that $(D(r) \mid p)=-1$, say $r=r^{\prime}$. Let $y_{1}=y_{1}^{\prime}=y_{1}\left(r^{\prime}\right)$ be the unique solution of

Then

$$
g_{r^{\prime}}\left(y_{1}\right) \equiv 0 .
$$

where

$$
r^{\prime} \equiv h\left(y_{1}^{\prime}\right)
$$

$$
h\left(y_{1}\right)=2^{-6} y_{1}^{-1}\left(64 b^{2}-16 a^{2} y_{1}-8 a y_{1}^{2}-y_{1}^{3}\right) .
$$

We note that $y_{1} \neq 0$ as $b \neq 0$. Now

$$
\begin{aligned}
n_{2} & =\frac{1}{4} \sum_{\substack{r \equiv h\left(y_{1}\right) \\
y_{1} \neq 0}}\left\{1-\left(\frac{D(r)}{p}\right)\right\}\left\{1+\left(\frac{y_{1}}{p}\right)\right\}+O(1) \\
& =\frac{1}{4} \sum_{y_{1} \neq 0}\left\{1-\left(\frac{y_{1}^{4} D\left(h\left(y_{1}\right)\right)}{p}\right)\right\}\left\{1+\left(\frac{y_{1}}{p}\right)\right\}+O(1) \\
& =\frac{p}{4}+O\left(p^{\frac{1}{1}}\right)-\frac{1}{4} \sum_{y_{1} \neq 0}\left(\frac{y_{1}^{4} D\left(h\left(y_{1}\right)\right)}{p}\right),
\end{aligned}
$$


by a deep result of Perel'muter [8] as

$$
y_{1}^{5} D\left(h\left(y_{1}\right)\right)
$$

is a polynomial of odd degree, namely 11 . The second sum is also $O\left(p^{\frac{1}{2}}\right)$ unless

$$
y^{4} D(h(y)) \equiv\{k(y)\}^{2} \quad(\bmod p),
$$

identically in $y$, where $k(y)$ is a quintic polynomial. (Note that the coefficient of $y^{10}$ on the left-hand side of (5.2) is $2^{-10}=\left(2^{-5}\right)^{2}$.) However it is easy to see that this is not so, since on taking $y=y_{1}^{\prime}$ we have

that is

$$
y_{1}^{\prime 4} D\left(h\left(y_{1}^{\prime}\right)\right) \equiv\left\{k\left(y_{1}^{\prime}\right)\right\}^{2},
$$

$$
y_{1}^{\prime 4} D\left(r^{\prime}\right) \equiv\left\{k\left(y_{1}^{\prime}\right)\right\}^{2},
$$

so that

$$
\left(\frac{D\left(r^{\prime}\right)}{p}\right)=+1 \text { or } 0,
$$

which is a contradiction. Hence we have proved

LEMMA 3.

$$
n_{2}= \begin{cases}\frac{1}{4}\left[1-\left(\frac{-1}{p}\right)\left\{1-\left(\frac{a^{2}}{p}\right)\right\}\right] p+O(1), & \text { if } \quad b \equiv 0, \\ \frac{1}{4} p+O\left(p^{\frac{1}{3}}\right), & \text { if } \quad b \neq 0 .\end{cases}
$$

Second proof. We note the obvious relation

$$
n_{1}+2 n_{2}+3 n_{3}+4 n_{4}=p .
$$

As we have evaluated $n_{1}$ and $n_{3}$, to determine $n_{2}$ (and $n_{4}$ ) it suffices to estimate

$$
n_{1}+4 n_{2}+9 n_{3}+16 n_{4} \text {. }
$$

We prove in an elementary way

Lemma 3'.

$$
n_{1}+4 n_{2}+9 n_{3}+16 n_{4}= \begin{cases}{\left[3+\left(\frac{-1}{p}\right)-\left(\frac{-a^{2}}{p}\right)\right] p+O(1),} & \text { if } \quad b \equiv 0, \\ 2 p+O\left(p^{\frac{1}{3}}\right), & \text { if } b \neq 0 .\end{cases}
$$

Proof.

$$
\begin{aligned}
\sum_{i=1}^{4} i^{2} n_{i} & =\sum_{i=0}^{4} \sum_{\substack{j=0 \\
N j=i}}^{p-1} i^{2}=\sum_{i=0}^{4} \sum_{\substack{j=0 \\
N j=i}}^{p-1} N_{j}^{2} \\
& =\sum_{j=0}^{p-1} N_{j}^{2}=N_{f},
\end{aligned}
$$


where $N_{f}$ denotes the number of solutions $(x, y)$ of

$$
f(x) \equiv f(y) \text {. }
$$

Let $N_{f}^{\prime}$ denote the number of such solutions with $x \neq 1$; then

$$
n_{1}+4 n_{2}+9 n_{3}+16 n_{4}=p+N_{f}^{\prime} \text {. }
$$

After cancelling the factor $x-y$ in (5.4) we find that solutions with $x$ ₹ $y$ satisfy

$$
(x+y)\left(x^{2}+y^{2}+a\right) \equiv-b .
$$

As there are at most three solutions of this with $x \equiv y$ we have

$$
N_{f}^{\prime}=N_{f}^{\prime \prime}+O(1)
$$

where $N_{f}^{\prime \prime}$ denotes the number of solutions $(x, y)$ of $(5.5)$. We now consider two cases according as $b \equiv 0$ or $b$ 丰 0 .

Case (i): $b \equiv 0$. Then (5.5) becomes

$$
(x+y)\left(x^{2}+y^{2}+a\right) \equiv 0
$$

and the number $N_{f}^{\prime \prime}$ of solutions $(x, y)$ of this is

$$
p+\left\{\left[1+\left(\frac{-1}{p}\right)-\left(\frac{-a^{2}}{p}\right)\right] p-\left(\frac{-1}{p}\right)\right\}-\left\{1+\left(\frac{-2 a}{p}\right)\right\}=\left\{2+\left(\frac{-1}{p}\right)-\left(\frac{-a^{2}}{p}\right)\right\} p+O(1)
$$

Case (ii): $b \neq 0$. Let $N_{k}^{\prime \prime}(1 \leqq k \leqq p-1)$ denote the number of solutions $(x, y)$ of the pair of congruences

Then

$$
x^{2}+y^{2}+a \equiv k, \quad x+y \equiv-b k^{-1} .
$$

$$
N_{f}^{\prime \prime}=\sum_{k=1}^{p-1} N_{k}^{\prime \prime}
$$

Eliminating $y$ from the pair (5.6), we find that $N_{k}^{\prime \prime}$ is just the number of solutions $x$ of

$$
x^{2}+b k^{-1} x+2^{-1}\left(b^{2} k^{-2}-k+a\right) \equiv 0 \text {. }
$$

Hence

$$
N_{k}^{\prime \prime}=1+\left(\frac{b^{2} k^{-2}-4.2^{-1}\left(b^{2} k^{-2}-k+a\right)}{p}\right)=1+\left(\frac{2 k^{3}-2 a k^{2}-b^{2}}{p}\right)
$$

and so

$$
N_{f}^{\prime \prime}=p-1+\sum_{k \neq 0}\left(\frac{2 k^{3}-2 a k^{2}-b^{2}}{p}\right) \text {. }
$$

As $b \neq 0$, by Manin's results [5],

$$
N_{f}^{\prime \prime}=p+O\left(p^{\frac{1}{2}}\right)
$$

This completes the proof of the lemma. 
6. Estimation of $n_{4}$. This follows at once from Lemmas 1, 2 and 3, or $3^{\prime}$ and (5.3). We have

LEMma 4.

$$
n_{4}= \begin{cases}\frac{p}{24}+O\left(p^{\frac{1}{2}}\right), & \text { if } \quad b \neq 0, \\ \frac{1}{8}\left[1+\left(\frac{-1}{p}\right)\left\{1-\left(\frac{a^{2}}{p}\right)\right\}\right] p+O(1), & \text { if } \quad b \equiv 0 .\end{cases}
$$

7. The number of residues in a complete residue system. The number of residues $N(f)=n_{1}+n_{2}+n_{3}+n_{4}$ of the quartic polynomial (2.2) (and so of $f_{1}(x)$ ) is given by

THEOREM 1.

$$
N(f)= \begin{cases}\frac{1}{4}+O(1), & \text { if } \quad a, b \equiv 0, p \equiv 1(\bmod 4), \\ \frac{1}{2} p+O(1), & \text { if } a, b \equiv 0, p \equiv 3(\bmod 4), \\ \frac{3}{8} p+O(1), & \text { if } a \neq 0, b \equiv 0, \\ \frac{5}{8} p+O\left(p^{\frac{1}{1}}\right), & \text { if } \quad b \neq 0 .\end{cases}
$$

In the cases where the error terms are $O(1)$, it would be very easy to prove exact results. In fact, quoting some results of R. D. von Sterneck [11], we have in these cases

$$
N(f)= \begin{cases}\frac{p+3}{4} & \text { for } a, b \equiv 0, p \equiv 1(\bmod 4), \\ \frac{p+1}{2} & \text { for } a, b \equiv 0, p \equiv 3(\bmod 4), \\ \frac{1}{8}\left(3 p+4-2\left(\frac{-a}{p}\right)+\left(\frac{-1}{p}\right)+2\left(\frac{-2 a}{p}\right)\right) & \text { for } a \neq 0, b \equiv 0 .\end{cases}
$$

8. Estimation of $m_{3}$. As $m_{3} \leqq n_{3}$ we have, from Lemma 1,

LEMMA 5.

$$
m_{3}=O(1)
$$

9. Estimation of $m_{1}$. If $b \equiv 0$, obviously $m_{1}=O(1)$, and so we may suppose that $b \neq 0$. As in $\$ 4$ we have

or equivalently

$$
m_{1}=\underset{\substack{r \\ \theta_{r} \operatorname{irred}(\bmod p)}}{\sum^{\prime} 1}+O(1)
$$

$$
m_{1}=h-\underset{\substack{r \\ g_{r} \operatorname{red}(\bmod p)}}{\sum^{\prime} 1}+O(1)
$$


Define $m^{(i)}(i=0,1,2,3)$ by

$$
m^{(i)}=\sum_{\substack{r \\ N_{r}=i}} 1
$$

where $\bar{N}$, denotes the number of solutions $y$ of $g_{r}(y) \equiv 0$, so that

$$
m_{1}=h-\left(m^{(1)}+m^{(3)}\right)+O(1) .
$$

Corresponding to (4.2) we prove that

We have

$$
m^{(1)}+3 m^{(3)}=h+O\left(p^{\frac{1}{2}} \log p\right) .
$$

$$
\begin{aligned}
\sum_{i=1}^{3} i m^{(i)} & =\sum_{i=0}^{3} \sum_{\substack{\tilde{N}_{r}=i \\
r}}^{\prime} i=\sum_{i=0}^{3} \sum_{\substack{\tilde{N}_{r}=i \\
r}}^{\prime} \tilde{N}_{r}=\sum_{r}^{\prime} \tilde{N}_{r} \\
& =(1 / p) \sum_{r}^{\prime} \sum_{y} \sum_{i} e\left(t g_{r}(y)\right) \\
& =h+(1 / p) \sum_{t \neq 0}\left\{\sum_{y} e\left(t\left(y^{3}+8 a y^{2}+16 a^{2} y-64 b^{2}\right)\right) \sum_{r}^{\prime} e(64 t y r)\right\} \\
& =h+(1 / p) \sum_{t \neq 0}\left\{\sum_{y \neq 0} e\left(t\left(y^{3}+8 a y^{2}+16 a^{2} y-64 b^{2}\right)\right) \sum_{r}^{\prime} e(64 t y r)\right\}+O(1)
\end{aligned}
$$

as $b \neq 0$. Now change the summation in $y$ to summation in $z$ defined by $z \equiv t y$, for fixed $t$. Then

$$
\begin{aligned}
\sum_{i=1}^{3} i m^{(i)}-h & =(1 / p) \sum_{t \neq 0}\left\{\sum_{z \neq 0} e\left(t^{-2} z^{3}+8 a t^{-1} z^{2}+16 a^{2} z-64 b^{2} t\right) \sum_{r}^{\prime} e(64 z r)\right\}+O(1) \\
& =(1 / p) \sum_{z \neq 0} e\left(16 a^{2} z\right)\left\{\sum_{t \neq 0} e\left(t^{-2} z^{3}+8 a t^{-1} z^{2}-64 b^{2} t\right)\right\}\left\{\sum_{r}^{\prime} e(64 z r)\right\}+O(1),
\end{aligned}
$$

and so

$$
\begin{aligned}
\left|\sum_{i=1}^{3} i m^{(i)}-h\right| & \leqq(1 / p) \sum_{z \neq 0}\left|\sum_{t \neq 0} e\left(t^{-2} z^{3}+8 a t^{-1} z^{2}-64 b^{2} t\right)\right|\left|\sum_{r}^{\prime} e(64 z r)\right|+O(1) \\
& \leqq(1 / p) \max _{1 \leqq z \leqq p-1}\left|\sum_{t \neq 0} e\left(z^{3} t^{-2}+8 a z^{2} t^{-1}-64 b^{2} t\right)\right| \sum_{z \neq 0}\left|\sum_{r} e(64 z r)\right|+O(1) .
\end{aligned}
$$

Now

and so

$$
\left|\sum_{r}^{\prime} e(64 z r)\right|=\left|\frac{1-e(64 z h m)}{1-e(64 z m)}\right| \leqq \frac{1}{|\sin (64 \pi z m / p)|}
$$

$$
\begin{aligned}
\sum_{z \neq 0}\left|\sum_{r}^{\prime} e(64 z r)\right| & \leqq \sum_{z=1}^{p-1} \frac{1}{|\sin (64 \pi z m / p)|}=\sum_{u=1}^{p-1} \frac{1}{\sin (\pi u / p)} \\
& =2 \sum_{u=1}^{\frac{1}{2}(p-1)} \frac{1}{\sin (\pi u / p)} \leqq p \sum_{u=1}^{\frac{1}{2}(p-1)}(1 / u) \\
& \leqq p \log p
\end{aligned}
$$


for $p$ large enough. Hence

$$
\left|\sum_{i=1}^{3} i m^{(i)}-h\right| \leqq \log p . \max _{1 \leqq z \leqq p-1}\left|\sum_{t \neq 0} e\left\{\frac{z^{3}+8 a z^{2} t-64 b^{2} t^{3}}{t^{2}}\right\}\right|+O(1)=O\left(p^{\frac{1}{2}} \log p\right),
$$

by a deep result of Perel'muter [8]. Now $m^{(2)}=O(1)$, so that

$$
m^{(1)}+3 m^{(3)}=h+O\left(p^{\frac{1}{2}} \log p\right) .
$$

Hence from (9.1) and (9.2) we have

$$
m_{1}=\frac{2}{3} h-\frac{2}{3} m^{(1)}+O\left(p^{\frac{1}{2}} \log p\right) .
$$

Now $g_{r}(y)$ has exactly one linear factor if and only if $(D(r) \mid p)=-1$. Hence

$$
m^{(1)}=\frac{1}{2} \sum_{r}^{\prime}\left\{1-\left(\frac{D(r)}{p}\right)\right\}+O(1)
$$

It is well-known that the above incomplete sum is $O\left(p^{\frac{1}{3}} \log p\right)$, so that

giving

$$
m^{(1)}=\frac{1}{2} h+O\left(p^{\frac{1}{2}} \log p\right)
$$

LEMMA 6.

$$
m_{1}=\left\{\begin{array}{lll}
\frac{1}{3} h+O\left(p^{\frac{1}{2}} \log p\right), & \text { if } & b \neq 0, \\
O(1), & \text { if } \quad b \equiv 0 .
\end{array}\right.
$$

10. Estimation of $m_{2}$. We consider two cases according as $b \equiv 0$ or $b \neq 0$.

Case $(i), b \equiv 0$. In this case, from $\S 5$, we have

$$
\begin{aligned}
m_{2} & =\frac{1}{4} \sum_{r}^{\prime}\left\{1-\left(\frac{-r}{p}\right)\right\}\left\{1+\left(\frac{4 r+a^{2}}{p}\right)\right\}+O(1) \\
& =\frac{1}{4}\left\{h+\sum_{r}^{\prime}\left(\frac{4 r+a^{2}}{p}\right)-\left(\frac{-1}{p}\right) \sum_{r}^{\prime}\left(\frac{r}{p}\right)-\left(\frac{-1}{p}\right) \sum_{r}^{\prime}\left(\frac{4 r^{2}+a^{2} r}{p}\right)\right\}+O(1) .
\end{aligned}
$$

The first two incomplete sums in $r$ are $O\left(p^{\frac{1}{2}} \log p\right)$ and the third one is also, unless $a \equiv 0$, when its sum is $h$. Hence

$$
m_{2}=\frac{1}{4}\left\{1-\left(\frac{-1}{p}\right)\left[1-\left(\frac{a^{2}}{p}\right)\right]\right\} h+O\left(p^{\frac{1}{4}} \log p\right) .
$$


Case (ii), $b$ ₹ 0 . Again from $\$ 5$ we have

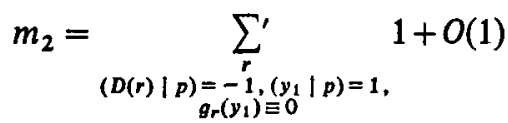

$$
\begin{aligned}
& =\frac{1}{4} \sum_{\substack{r \\
r=h\left(y_{1}\right)}}^{\prime} \sum_{y_{1} \neq 0}\left\{1+\left(\frac{D(r)}{p}\right)\right\}\left\{1+\left(\frac{y_{1}}{p}\right)\right\}+O(1) \\
& =\frac{1}{4 p} \sum_{\substack{r \equiv h\left(y_{1}\right) \\
y_{1} \neq 0}}\left\{1-\left(\frac{D(r)}{p}\right)\right\}\left\{1+\left(\frac{y_{1}}{p}\right)\right\} \sum_{s}^{\prime} \sum_{t} e(t(r-s))+O(1) \\
& =\frac{h}{4 p} \sum_{r \equiv h\left(y_{1}\right)} \sum_{y_{1} \neq 0}\left\{1-\left(\frac{D(r)}{p}\right)\right\}\left\{1+\left(\frac{y_{1}}{p}\right)\right\} \\
& +\frac{1}{4 p} \sum_{t \neq 0}\left\{\sum_{r \equiv h\left(y_{1}\right)} \sum_{y_{1} \neq 0}\left\{1-\left(\frac{D(r)}{p}\right)\right\}\left\{1+\left(\frac{y_{1}}{p}\right)\right\} e(t r) \sum_{s}^{\prime} e(-s t)\right\}+O(1) .
\end{aligned}
$$

Hence

$\left|m_{2}-\frac{h}{p} n_{2}\right| \leqq \frac{1}{4 p} \max _{1 \leqq t \leqq p-1}\left|\sum_{y_{1} \neq 0}\left\{1-\left(\frac{D\left(h\left(y_{1}\right)\right)}{p}\right)\right\}\left\{1+\left(\frac{y_{1}}{p}\right)\right\} e\left(t h\left(y_{1}\right)\right)\right| \sum_{t \neq 0}\left|\sum_{s}^{\prime} e(-s t)\right|+O(1)$

and so from a deep result of Perel'muter [8]

$$
m_{2}=\frac{h n_{2}}{p}+O\left(p^{\frac{1}{2}} \log p\right)=\frac{h}{4}+O\left(p^{\frac{1}{2}} \log p\right) .
$$

We have proved

\section{LEMMA 7.}

$$
m_{2}= \begin{cases}\frac{1}{4}\left\{1-\left(\frac{-1}{p}\right)\left[1-\left(\frac{a^{2}}{p}\right)\right]\right\} h+O\left(p^{\frac{1}{2}} \log p\right), & \text { if } b \equiv 0, \\ \frac{h}{4}+O\left(p^{\frac{1}{2}} \log p\right), & \text { if } b \neq 0 .\end{cases}
$$

11. Estimation of $m_{4}$. It is easy to show in a similar (but easier) way to that used in the proof of

in $\$ 9$, that

$$
m^{(1)}+2 m^{(2)}+3 m^{(3)}=h+O\left(p^{\frac{1}{2}} \log p\right)
$$

$$
m_{1}+2 m_{2}+3 m_{3}+4 m_{4}=h+O\left(p^{\frac{1}{3}} \log p\right) .
$$


Hence, from Lemmas 5, 6 and 7, we have

Lemma 8.

$$
m_{4}= \begin{cases}\frac{h}{24}+O\left(p^{\frac{1}{2}} \log p\right), & \text { if } \quad b \neq 0 \\ \frac{1}{8}\left\{1+\left(\frac{-1}{p}\right)\left[1-\left(\frac{a^{2}}{p}\right)\right]\right\} h+O\left(p^{\frac{1}{2}} \log p\right), & \text { if } \quad b=0 .\end{cases}
$$

12. The number of residues in an arithmetic progression. The number of residues $M(f)=m_{1}+m_{2}+m_{3}+m_{4}$ of the quartic polynomial (2.11), and so of (2.1), in the arithmetic progression (2.12) is given by

THEOREM 2.

$$
M(f)=\left\{\begin{array}{lll}
\frac{1}{4} h+O\left(p^{\frac{1}{2}} \log p\right), & \text { if } a, b \equiv 0, p \equiv 1(\bmod 4), \\
\frac{1}{2} h+O\left(p^{\frac{1}{2}} \log p\right), & \text { if } a, b \equiv 0, p \equiv 3(\bmod 4), \\
\frac{3}{8} h+O\left(p^{\frac{1}{2}} \log p\right), & \text { if } a \neq 0, b \equiv 0, \\
\frac{5}{8} h+O\left(p^{\frac{1}{2}} \log p\right), & \text { if } b \neq 0 .
\end{array}\right.
$$

13. Some corollaries of Theorem 2. By choosing $h$ large enough in the asymptotic formulae of Theorem 2 we can guarantee that $M(f)>0$. This proves

THEOREM 3. Any arithmetic progression with $\gg p^{\frac{1}{2}} \log p$ terms contains a residue and non-residue $(\bmod p)$ of $f(x)$.

We also note that Theorem 2 implies

THeOREM 4. If $b \neq 0$, any arithmetic progression with $\gg p^{\frac{1}{2}} \log p$ terms contains a pair of consecutive residues $(\bmod p)$ of $f(x)$.

Proof. As $b$ 丰 0 , by Theorem 2,

$$
M(f)=\frac{5}{8} h+O\left(p^{\frac{1}{2}} \log p\right) .
$$

Hence, for all $p \geqq p_{0}$, there exists a constant $k>0$ such that

Choose

$$
M(f)>\frac{5}{8} h-k p^{\frac{1}{2}} \log p .
$$

so that

$$
h=\left[9 k p^{\frac{1}{2}} \log p\right]+1,
$$

$$
M(f)>\frac{37}{8} k p^{\frac{1}{1}} \log p>0 .
$$

We show that

$$
l, l+m, l+2 m, \ldots, l+(h-1) m,
$$


with this value of $h$, always contains a pair of consecutive residues. For suppose not; then

and so, for $p \geqq p_{0}$,

$$
M(f) \leqq\left[\frac{h}{2}\right]+1
$$

$$
\frac{5}{8} h-k p^{\frac{1}{2}} \log p \leqq \frac{1}{2} h+1 \text {, }
$$

which implies, for large enough $p$, the contradiction

$$
h \leqq 8 k p^{\frac{1}{2}} \log p+8
$$

We remark that a number of other results, similar to Theorems 3 and 4, can be obtained in much the same way and that most of the results of this paper, with only slight modifications, go over to quartics over a general finite field.

14. The least pair of consecutive residues when $b \equiv 0$. When $b \equiv 0$, the asymptotic formulae of Theorem 2 tell us that there are far fewer residues of $f(x)(\bmod p)$, and we do not have enough information to guarantee the existence of a pair of consecutive ones in this case. To overcome this difficulty we determine asymptotic formulae for the number $\mathfrak{M}$ of pairs of consecutive residues in the arithmetic progression (2.3). To do this we set

$$
m_{i j}=\sum_{\substack{r \\ N_{r}=i, N_{r+m}=j}}^{\prime} 1 \quad(i, j=0,1,2,3,4)
$$

so that

Now it is clear that

$$
\mathfrak{m}=\sum_{i, j=1}^{4} m_{i j}
$$

and

$$
m_{13}, m_{23}, m_{31}, m_{32}, m_{33}, m_{34}, m_{43} \leqq m_{3}
$$

$$
m_{11}, m_{12}, m_{14}, m_{24}, m_{41} \leqq m_{1}
$$

hence by Lemmas 5 and 6 we have

LeMmA 9. When $b \equiv 0$, each of $m_{11}, m_{12}, m_{13}, m_{14}, m_{21}, m_{23}, m_{31}, m_{32}, m_{33}, m_{34}, m_{43}$ is $O(1)$.

Thus (14.2) becomes

$$
\mathfrak{M}=m_{22}+m_{24}+m_{42}+m_{44}+O(1)
$$

so that we are left with the problem of estimating $m_{22}, m_{24}, m_{42}$ and $m_{44}$. We begin with $m_{22}$. 
LEMma 10. When $b \equiv 0$,

Proof. Appealing to Carlitz's results [2] we see that

$$
m_{22}=\left\{\begin{aligned}
\frac{1}{8}\left\{1-\left(\frac{-1}{p}\right)\right\} h+O\left(p^{\frac{1}{1}} \log p\right), & \text { if } a \equiv 0, \\
\frac{1}{16}\left\{1-\left(\frac{-1}{p}\right)\right\} h+O\left(p^{\frac{1}{2}} \log p\right), & \text { if } a^{2} \equiv \pm 4 m, \\
\frac{1}{16} h+O\left(p^{\frac{1}{1}} \log p\right), & \text { otherwise. }
\end{aligned}\right.
$$

$$
x^{4}+a x^{2}-r
$$

is congruent $(\bmod p)$ to the product of two distinct linear factors and an irreducible quadratic if and only if

$$
\left(\frac{-r}{p}\right)=-1 \text { and }\left(\frac{4 r+a^{2}}{p}\right)=+1 \text {. }
$$

For convenience we set $a \equiv 2 c$ so that the second condition of (14.4) becomes $\left(r+c^{2} \mid p\right)=+1$. Hence

where, in the summation,

$$
m_{22}=\sum_{r}^{\prime} 1+O(1)
$$

Hence

$$
\left(\frac{-r}{p}\right)=-1, \quad\left(\frac{r+c^{2}}{p}\right)=+1, \quad\left(\frac{-(r+m)}{p}\right)=-1, \quad\left(\frac{r+\left(m+c^{2}\right)}{p}\right)=+1 .
$$

$$
m_{22}=\frac{1}{16} \sum_{r}^{\prime}\left\{1-\left(\frac{-r}{p}\right)\right\}\left\{1-\left(\frac{-(r+m)}{p}\right)\right\}\left\{1+\left(\frac{r+c^{2}}{p}\right)\right\}\left\{1+\left(\frac{r+\left(m+c^{2}\right)}{p}\right)\right\}+O(1) .
$$

Now unless, after multiplying the expressions in the four brackets together, we obtain squares in the Legendre symbols, this gives

$$
m_{22}=\frac{1}{16} h+O\left(p^{\frac{1}{2}} \log p\right)
$$

Now squares occur if and only if one of the following three possibilities holds: (i) $c \equiv 0$, (ii) $c^{2} \equiv m$, (iii) $c^{2} \equiv-m$.

If (i) holds,

$$
\begin{aligned}
m_{22} & =\frac{1}{16} \sum_{r}^{\prime}\left\{1-\left(\frac{-r}{p}\right)\right\}\left\{1+\left(\frac{r}{p}\right)\right\}\left\{1-\left(\frac{-(r+m)}{p}\right)\right\}\left\{1+\left(\frac{r+m}{p}\right)\right\}+O(1) \\
& =\frac{1}{16} \sum_{r}^{\prime}\left\{1-\left(\frac{-1}{p}\right)\right\}\left\{1+\left(\frac{r}{p}\right)\right\}\left\{1-\left(\frac{-1}{p}\right)\right\}\left\{1+\left(\frac{r+m}{p}\right)\right\}+O(1) \\
& =\frac{1}{16}\left\{1-\left(\frac{-1}{p}\right)\right\}^{2} \sum_{r}^{\prime}\left\{1+\left(\frac{r}{p}\right)\right\}\left\{1+\left(\frac{r+m}{p}\right)\right\}+O(1) \\
& =\frac{1}{8}\left\{1-\left(\frac{-1}{p}\right)\right\} h+O\left(p^{\frac{1}{2}} \log p\right) .
\end{aligned}
$$


Similarly if (ii) or (iii) holds we have

$$
m_{22}=\frac{1}{16}\left\{1-\left(\frac{-1}{p}\right)\right\} h+O\left(p^{\frac{1}{2}} \log p\right) .
$$

This completes the proof of Lemma 10.

LEMMA 11. When $b \equiv 0$,

$$
m_{24}= \begin{cases}O(1), & \text { if } a \equiv 0, \\ \left\{1+\left(\frac{-1}{p}\right)\right\} \frac{h}{32}+O\left(p^{\frac{1}{2}} \log p\right), & \text { if } a^{2} \equiv 4 m, \\ \left\{1-\left(\frac{-1}{p}\right)\right\} \frac{h}{32}+O\left(p^{\frac{1}{2}} \log p\right), & \text { if } a^{2} \equiv-4 m, \\ \frac{h}{32}+O\left(p^{\frac{1}{2}} \log p\right), & \text { otherwise. }\end{cases}
$$

Proof. From Lemma 3 , when $a, b \equiv 0$ and $p \equiv 1(\bmod 4)$,

$$
n_{2}=O(1) \text {. }
$$

As $m_{24} \leqq m_{2} \leqq n_{2}$, we have $m_{24}=O(1)$. From Lemma 4 when $a, b \equiv 0$ and $p \equiv 3(\bmod 4)$,

$$
n_{4}=O(1)
$$

As $m_{24} \leqq m_{4} \leqq n_{4}$, we have $m_{24}=O(1)$.

Hence we may suppose that $a \neq 0$. From Carlitz's result we have that $x^{4}+a x^{2}-r$ is congruent $(\bmod p)$ to the product of two distinct linear factors and an irreducible quadratic if and only if

$$
\left(\frac{-r}{p}\right)=-1 \text { and }\left(\frac{r+c^{2}}{p}\right)=+1
$$

where $a \equiv 2 c$; also

$$
y^{4}+a y^{2}-(r+m)
$$

is congruent $(\bmod p)$ to the product of four distinct linear factors if and only if

and

$$
\left(\frac{-(r+m)}{p}\right)=+1, \text { say } r+m \equiv-s^{2} \text {, }
$$

Hence

$$
\left(\frac{c^{2}-s^{2}}{p}\right)=+1, \quad\left(\frac{-2(c+s)}{p}\right)=+1 .
$$

$$
m_{24}=\sum_{s=1}^{\frac{1}{2}(p-1)} \sum_{r}^{\prime} 1+O(1)
$$


where, in the summations, $r+m \equiv-s^{2}$ and

Setting

$$
\left(\frac{-r}{p}\right)=-1,\left(\frac{r+c^{2}}{p}\right)=+1,\left(\frac{-2(c+s)}{p}\right)=+1, \quad\left(\frac{c^{2}-s^{2}}{p}\right)=+1 .
$$

and

$$
A(r, s)=\left\{1-\left(\frac{-r}{p}\right)\right\}\left\{1+\left(\frac{r+c^{2}}{p}\right)\right\}\left\{1+\left(\frac{-2(c+s)}{p}\right)\right\}\left\{1+\left(\frac{c^{2}-s^{2}}{p}\right)\right\}
$$

for convenience, we have

$$
B(s)=A\left(-s^{2}-m, s\right)
$$

$$
\begin{aligned}
& m_{24}=\frac{1}{16} \sum_{\substack{s=1 \\
r+m=-s^{2}}}^{ \pm(p-1)} \sum_{\substack{r \\
r}}^{\prime} A(r, s)+O(1) \\
& =\frac{1}{32} \sum_{\substack{s \\
r+m=-s^{2}}} \sum_{\substack{r \\
r+m}}^{\prime} A(r, s)+O(1) \\
& =\frac{1}{32} \sum_{\substack{s \\
r+m \equiv-s^{2}}} \sum_{\substack{r \\
\prime}}^{\prime} A(r, s) \sum_{u}^{\prime} \sum_{r} e(t(u-r))+O(1) \\
& =\frac{h}{32 p} \sum_{\substack{s, r \\
r+m=-s^{2}}} A(r, s)+\frac{1}{32 p} \sum_{t \neq 0}\left\{\sum_{\substack{s, r \\
r+m \equiv-s^{2}}} A(r, s) e(-t r)\right\}\left\{\sum_{u}^{\prime} e(t u)\right\}+O(1) \\
& =\frac{h}{32 p} \sum_{s} B(s)+\frac{1}{32 p} \sum_{t \neq 0}\left\{\sum_{s} B(s) e\left(\left(s^{2}+m\right) t\right)\right\}\left\{\sum_{u}^{\prime} e(t u)\right\}+O(1) .
\end{aligned}
$$

Hence

$$
\begin{aligned}
\left|m_{24}-\frac{h}{32 p} \sum_{s} B(s)\right| & \leqq \frac{1}{32 p} \max _{1 \leqq t \leqq p-1}\left|\sum_{s} B(s) e\left(\left(s^{2}+m\right) t\right)\right| \sum_{t \neq 0}\left|\sum_{u}^{\prime} e(t u)\right|+O(1) \\
& =O\left(p^{\frac{1}{2}} \log p\right),
\end{aligned}
$$

by a result of Perel'muter [8]. We now consider

$$
\sum_{s}\left\{1-\left(\frac{s^{2}+m}{p}\right)\right\}\left\{1+\left(\frac{-s^{2}+\left(c^{2}-m\right)}{p}\right)\right\}\left\{1+\left(\frac{-2(s+c)}{p}\right)\right\}\left\{1+\left(\frac{-s^{2}+c^{2}}{p}\right)\right\} .
$$

By Perel'muter's results this is

$$
p+O\left(p^{\frac{1}{2}}\right)
$$

except in a few special cases. Thus in general

$$
m_{24}=\frac{h}{32}+O\left(p^{\frac{1}{2}} \log p\right) .
$$


As $c, m \neq 0$ the special cases are easily seen to arise when

When $c^{2} \equiv m,(14.5)$ becomes

$$
c^{2} \equiv m \quad \text { or } \quad c^{2} \equiv-m
$$

$$
\begin{aligned}
\sum_{s}\left\{1-\left(\frac{s^{2}+c^{2}}{p}\right)\right\} & \left\{1+\left(\frac{-s^{2}}{p}\right)\right\}\left\{1+\left(\frac{-2(s+c)}{p}\right)\right\}\left\{1+\left(\frac{-s^{2}+c^{2}}{p}\right)\right\} \\
& =\sum_{s}\left\{1+\left(\frac{-1}{p}\right)\right\}\left\{1-\left(\frac{s^{2}+c^{2}}{p}\right)\right\}\left\{1+\left(\frac{-2(s+c)}{p}\right)\right\}\left\{1+\left(\frac{-s^{2}+c^{2}}{p}\right)\right\}+O(1) \\
& =\left\{1+\left(\frac{-1}{p}\right)\right\} p+O\left(p^{\frac{1}{2}}\right),
\end{aligned}
$$

giving

$$
m_{24}=\left\{1+\left(\frac{-1}{p}\right)\right\} \frac{h}{32}+O\left(p^{\frac{1}{2}} \log p\right) .
$$

Similarly, when $c^{2} \equiv-m$, we obtain

$$
m_{24}=\left\{1-\left(\frac{-1}{p}\right)\right\} \frac{h}{32}+O\left(p^{\frac{1}{2}} \log p\right) .
$$

This completes the proof of Lemma 11. In an almost identical way we can prove

LEMMA 12. When $b \equiv 0$,

$$
m_{42}=\left\{\begin{array}{cl}
O(1), & \text { if } a \equiv 0 \\
\left\{1-\left(\frac{-1}{p}\right)\right\} \frac{h}{32}+O\left(p^{\frac{1}{2}} \log p\right), & \text { if } a^{2} \equiv 4 m \\
\left\{1+\left(\frac{-1}{p}\right)\right\} \frac{h}{32}+O\left(p^{\frac{1}{2}} \log p\right), & \text { if } a^{2} \equiv-4 m \\
\frac{h}{32}+O\left(p^{\frac{1}{2}} \log p\right), & \text { otherwise. }
\end{array}\right.
$$

Finally we evaluate $m_{44}$.

LEMMA 13. When $b \equiv 0$,

$$
m_{44}=\left\{\begin{aligned}
\left\{1+\left(\frac{-1}{p}\right)\right\} \frac{h}{32}+O\left(p^{\frac{1}{2}} \log p\right), & \text { if } a \equiv 0 \\
\left\{1+\left(\frac{-1}{p}\right)\right\} \frac{h}{64}+O\left(p^{\frac{1}{2}} \log p\right), & \text { if } a^{2} \equiv \pm 4 m \\
\frac{h}{64}+O\left(p^{\frac{1}{2}} \log p\right), & \text { otherwise. }
\end{aligned}\right.
$$


Proof. As $x^{4}+a x^{2}-r$ is congruent $(\bmod p)$ to the product of four distinct linear factors if and only if

and

$$
\left(\frac{-r}{p}\right)=+1, \text { say } r \equiv-s^{2},
$$

we have

$$
\left(\frac{c^{2}-s^{2}}{p}\right)=+1, \quad\left(\frac{-2(c+s)}{p}\right)=+1,
$$

Hence

$$
m_{44}=\sum_{t=1}^{\frac{1}{2}(p-1)} \sum_{s=1}^{\frac{1}{2}(p-1)} \sum_{r}^{\prime} 1 \text {, }
$$

where, in the summations, $r \equiv-s^{2}, r+m \equiv-t^{2}$, and

$$
\left(\frac{c^{2}-s^{2}}{p}\right)=+1,\left(\frac{-2(c+s)}{p}\right)=+1, \quad\left(\frac{c^{2}-t^{2}}{p}\right)=+1, \quad\left(\frac{-2(c+t)}{p}\right)=+1 .
$$

$$
\begin{aligned}
m_{44}=\frac{1}{16} \sum_{\substack{t=1 \\
r \equiv-s^{2}, s^{2}-t^{2} \equiv m}}^{t(p-1)} \sum_{r}^{\frac{1}{2}(p-1)}\left\{1+\left(\frac{c^{2}-s^{2}}{p}\right)\right\}\left\{1+\left(\frac{-2(c+s)}{p}\right)\right\}\left\{1+\left(\frac{c^{2}-t^{2}}{p}\right)\right\} \\
\times\left\{1+\left(\frac{-2(c+t)}{p}\right)\right\}+O(1) . \\
=\frac{1}{64} \sum_{\substack{t, s \\
r \equiv-s^{2}, s^{2}-t^{2} \equiv m}}\left\{1+\left(\frac{c^{2}-s^{2}}{p}\right)\right\}\left\{1+\left(\frac{-2(c+s)}{p}\right)\right\}\left\{1+\left(\frac{c^{2}-t^{2}}{p}\right)\right\} \\
\times\left\{1+\left(\frac{-2(c+t)}{p}\right)\right\}+O(1) .
\end{aligned}
$$

Now change the summation over $s$ and $t$ to one over $u$ and $t$, where $u$ is defined by

$$
s \equiv t+u \text {. }
$$

\section{Hence}

$$
\begin{aligned}
& m_{44}=\frac{1}{64} \sum_{\substack{u, t \\
r=(t) \\
u^{2}+2 u t-m=0}}^{\prime}\left\{1+\left(\frac{c^{2}-(t+u)^{2}}{p}\right)\right\}\left\{1+\left(\frac{-2(c+t+u)}{p}\right)\right\}\left\{1+\left(\frac{c^{2}-t^{2}}{p}\right)\right\} \\
& \times\left\{1+\left(\frac{-2(c+t)}{p}\right)\right\}+O(1) \\
& =\frac{1}{64} \sum_{\substack{u \neq 0 \\
4 u^{2} r=-\left(m+u^{2}\right)^{2}}}\left\{1+\left(\frac{c^{2}-\left(m+u^{2}\right)^{2} / 4 u^{2}}{p}\right)\right\}\left\{1+\left(\frac{-2\left(c+\left(m+u^{2}\right) / 2 u\right)}{p}\right)\right\} \\
& \times\left\{1+\left(\frac{c^{2}-\left(m-u^{2}\right)^{2} / 4 u^{2}}{p}\right)\right\}\left\{1+\left(\frac{-2\left(c+\left(m-u^{2}\right) / 2 u\right)}{p}\right)\right\}+O(1) \\
& =\frac{1}{64} \sum_{\substack{u \neq 0 \\
4 u^{2} r=-\left(m+u^{2}\right)^{2}}} \sum_{\substack{\prime \\
r}} C(u)+O(1) \text {, }
\end{aligned}
$$


where

$$
\begin{aligned}
C(u)=\left\{1+\left(\frac{-u^{4}+\left(4 c^{2}-2 m\right) u^{2}-m^{2}}{p}\right)\right\}\left\{1+\left(\frac{-u^{3}-2 c u^{2}-m u}{p}\right)\right\} & \\
\times & \left\{1+\left(\frac{-u^{4}+\left(4 c^{2}+2 m\right) u^{2}-m^{2}}{p}\right)\right\}\left\{1+\left(\frac{u^{3}-2 c u^{2}-m u}{p}\right)\right\} .
\end{aligned}
$$

Thus

$$
\begin{aligned}
m_{44} & =\frac{1}{64 p} \sum_{\substack{u \neq 0 \\
4 u^{2} r \equiv-\left(m+u^{2}\right)^{2}}} \sum_{r} C(u) \sum_{w}^{\prime} \sum_{r} e(t(w-r)+O(1) \\
& =\frac{h}{64 p} \sum_{\substack{u \neq 0 \\
4 u^{2} r \equiv-\left(m+u^{2}\right)^{2}}} C(u)+\frac{1}{64 p} \sum_{t \neq 0}\left\{\sum_{\substack{u \neq 0 \\
4 u^{2} r \equiv-\left(m+u^{2}\right)^{2}}} C(u) e(-r t)\right\}\left\{\sum_{w}^{\prime} e(t w)\right\}+O(1) \\
& =\frac{h}{64 p} \sum_{w \neq 0} C(u)+\frac{1}{64 p} \sum_{t \neq 0}\left\{\sum_{u \neq 0} C(u) e\left\{t\left(m+u^{2}\right)^{2} / 4 u^{2}\right\}\right\}\left\{\sum_{w}^{\prime} e(t w)\right\}+O(1)
\end{aligned}
$$

and so

$$
\begin{aligned}
\left|m_{44}-\frac{h}{64 p} \sum_{u \neq 0} C(u)\right| & \leqq\left|\frac{1}{64 p} \max _{1 \leqq t \leqq p-1}\right| \sum_{u \neq 0} C(u) e\left\{t\left(m+u^{2}\right)^{2} / 4 u^{2}\right\}\left|\sum_{t \neq 0} \sum_{w}{ }^{\prime} e(t w)\right|+O(1) \\
& =O\left(p^{\frac{1}{2}} \log p\right),
\end{aligned}
$$

by Perel'muter's results [8]. We must therefore consider

$$
\begin{aligned}
\sum_{u \neq 0}\left\{1+\left(\frac{-u^{4}+\left(4 c^{2}-2 m\right) u^{2}-m^{2}}{p}\right)\right\}\left\{1+\left(\frac{-u^{3}-2 c u^{2}-m u}{p}\right)\right\} \\
\times\left\{1+\left(\frac{-u^{4}+\left(4 c^{2}+2 m\right) u^{2}-m^{2}}{p}\right)\right\}\left\{1+\left(\frac{+u^{3}-2 c u^{2}-m u}{p}\right)\right\} .
\end{aligned}
$$

In general this is $p+O\left(p^{\frac{1}{2}}\right)$ except for a few special cases, and so

$$
m_{44}=\frac{h}{64}+O\left(p^{\frac{1}{2}} \log p\right)
$$

It is easy to check that the special cases only occur if $c \equiv 0, c^{2} \equiv m$ or $c^{2} \equiv-m$.

If $c \equiv 0$, (14.6) becomes

$$
\begin{aligned}
& \sum_{u \neq 0}\left\{1+\left(\frac{-\left(u^{2}+m\right)^{2}}{p}\right)\right\}\left\{1+\left(\frac{-u\left(u^{2}+m\right)}{p}\right)\right\}\left\{1+\left(\frac{-\left(u^{2}-m\right)^{2}}{p}\right)\right\}\left\{1+\left(\frac{u\left(u^{2}-m\right)}{p}\right)\right\} \\
& =\sum_{u \neq 0}\left\{1+\left(\frac{-1}{p}\right)\right\}^{2}\left\{1+\left(\frac{-u\left(u^{2}+m\right)}{p}\right)\right\}\left\{1+\left(\frac{u\left(u^{2}-m\right)}{p}\right)\right\}+O(1) \\
& =2\left\{1+\left(\frac{-1}{p}\right)\right\}\left\{p+O\left(p^{\frac{1}{2}}\right)\right\},
\end{aligned}
$$



so that

$$
m_{44}=\left\{1+\left(\frac{-1}{p}\right)\right\} \frac{h}{32}+O\left(p^{\frac{1}{2}} \log p\right) .
$$

If $c^{2} \equiv m,(14.6)$ becomes

$$
\begin{aligned}
\sum_{u \neq 0} & \left\{1+\left(\frac{-\left(u^{2}-c^{2}\right)^{2}}{p}\right)\right\}\left\{1+\left(\frac{-u(u+c)^{2}}{p}\right)\right\}\left\{1+\left(\frac{-u^{4}+6 c^{2} u^{2}-m^{2}}{p}\right)\right\}\left\{1+\left(\frac{u\left(u^{2}-2 c u-c^{2}\right)}{p}\right)\right\} \\
= & \sum_{u \neq 0}\left\{1+\left(\frac{-1}{p}\right)\right\}\left\{1+\left(\frac{-u(u+c)^{2}}{p}\right)\right\}\left\{1+\left(\frac{-u^{4}+6 c^{2} u^{2}-m^{2}}{p}\right)\right\} \\
= & \times\left\{1+\left(\frac{u\left(u^{2}-2 c u-c^{2}\right)}{p}\right)\right\}+O(1) \\
& \left\{1+\left(\frac{-1}{p}\right)\right\} p+O\left(p^{\frac{1}{2}}\right),
\end{aligned}
$$

and therefore

$$
m_{44}=\left\{1+\left(\frac{-1}{p}\right)\right\} \frac{h}{64}+O\left(p^{\frac{1}{2}} \log p\right)
$$

The case $c^{2} \equiv-m$ is exactly similar. This completes the proof of Lemma 13. Putting together the results of Lemmas 10,11, 12 and 13 we obtain (using 14.3)

THEOREM 5. If $b \equiv 0$,

$$
\mathbb{U}=\left\{\begin{array}{lll}
\frac{h}{16}+O\left(p^{\frac{1}{}} \log p\right), & \text { if } a \equiv 0, & p \equiv 1(\bmod 4), \\
\frac{h}{4}+O\left(p^{\frac{1}{2}} \log p\right), & \text { if } a \equiv 0, & p \equiv 3(\bmod 4), \\
\frac{3 h}{32}+O\left(p^{\frac{1}{2}} \log p\right), & \text { if } a^{2} \equiv 4 m, & p \equiv 1(\bmod 4), \\
\frac{3 h}{16}+O\left(p^{\frac{1}{2}} \log p\right), & \text { if } a^{2} \equiv 4 m, & p \equiv 3(\bmod 4), \\
\frac{3 h}{32}+O\left(p^{\frac{1}{2}} \log p\right), & \text { if } a^{2} \equiv-4 m, & p \equiv 1(\bmod 4), \\
\frac{3 h}{16}+O\left(p^{\frac{1}{2}} \log p\right), & \text { if } a^{2} \equiv-4 m, & p \equiv 3(\bmod 4), \\
\frac{9 h}{64}+O\left(p^{\frac{1}{2}} \log p\right), & \text { otherwise. }
\end{array}\right.
$$


An immediate corollary of this is

THEOREM 6. If $b \equiv 0$, any arithmetic progression with $\gg p^{\frac{1}{5}} \log p$ terms contains a pair of consecutive residues $(\bmod p)$ of $f(x)$.

15. A conjecture. We conclude this paper by making the following

Conjecture. The number $M(f)$ of residues $(\bmod p)$ of a general polynomial $f(x)$ of degree $d$ in an arithmetic progression of $h$ terms is given by

$$
M(f)=\lambda h+O\left(p^{\frac{1}{2}} \log p\right),
$$

where $\lambda$ is the constant given by Birch and Swinnerton-Dyer [1] and the constant implied by the $O$-symbol depends only on $d$.

We remark that it is true when $d=2,3$ or 4 .

\section{REFERENCES}

1. B. J. Birch and H. P. F. Swinnerton-Dyer, Note on a problem of Chowla, Acta Arith. 5 (1959), 417-423.

2. L. Carlitz, Note on a quartic congruence, Amer. Math. Monthly 63 (1956), 569-571.

3. L. Carlitz and S. Uchiyama, Bounds for exponential sums, Duke Math. J. 24 (1957), 37-41.

4. L. E. Dickson, Criteria for the irreducibility of functions in a finite field, Bull. Amer. Math. Soc. 13 (1906), 1-8. $1-7$.

5. Yu. I. Manin, On a cubic congruence to a prime modulus, Amer. Math. Soc. Transl. 13 (1960),

6. K. McCann and K. S. Williams, On the residues of a cubic polynomial, Canad. Math. Bull. 10 (1967), 29-38.

7. L. J. Mordell, On the least residue and non-residue of a polynomial, J. Lond. Math. Soc. 38 (1963), 451-453.

8. G. I. Perel'muter, On certain sums of characters, Uspehi Mat. Nauk. 18 (1963), 145-149.

9. Th. Skolem, The general congruence of 4 th degree modulo $p, p$ prime, Norske Mat. Tidsskr. 34 (1952), 73-80.

10. S. Uchiyama, Sur le nombre des valeurs distinctes d'un polynôme à coefficients dans un corps fini, Proc. Japan Acad. 30 (1954), 930-933.

11. R. D. Von Sterneck, Über die Anzahl inkongruenter Werte die eine ganze Function dritte Grades annimont, S.-B. Akad. Wiss. Wien. Math. Kl. 116 (1907), 895-904.

12. A. Weil, Sur les courbes algébriques et les variétés qui s'en déduisent, Actualités Sci. et Ind. 1041 (Paris, 1948).

13. K. S. Williams, Pairs of consecutive residues of polynomials, Canad. J. Math. 19 (1967), $655-666$

14. K. S. Williams, On the least non-residue of a quartic polynomial, Proc. Cambridge Phil. Soc. 62 (1966), 429-431. 